'Departamento de

Gastroenterología, Clínica Las Condes. Santiago, Chile.

2Programa Enfermedad

Inflamatoria Intestinal, Servicio de Gastroenterología, Clínica Las Condes. Santiago, Chile.

${ }^{3}$ Programa Enfermedad Inflamatoria Intestinal, Unidad de Coloproctología, Clínica Las

Condes. Santiago, Chile.

${ }^{4}$ Subdirección de Investigación, Dirección Académica, Clínica Las Condes. Santiago, Chile.

5Unidad de Inmunología, Servicio de Medina Interna, Clínica Las Condes. Santiago, Chile. aecada en Capacitación Bases para el Manejo de la Patología Intestinal. ${ }^{b}$ Enfermera.

'Fellow Programa Enfermedad Inflamatoria Intestinal, Departamento de Gastroenterología, Clínica Las Condes. Santiago, Chile.

Recibido el 6 de septiembre de 2016, aceptado el 15 de marzo de 2017.

Correspondencia a: Dr. Rodrigo Quera Departamento de Gastroenterología, Clínica Las Condes. Santiago, Chile. Estoril 450, Las Condes. Teléfono: 226108048 Fax: 226208719 rquera@clc.cl

\section{Experiencia local con natalizumab en pacientes con enfermedad de Crohn refractaria a anti-TNF. Casos clínicos}

\author{
DANIELA FLUXÁ ${ }^{1, \mathrm{a}}$, PATRICIO IBÁÑEZ ${ }^{2}$, LILIAN FLORES ${ }^{2, \mathrm{a}}$, \\ CAROLINA FIGUEROA ${ }^{2}$, JAIME LUBASCHER ${ }^{2}$, \\ UDO KRONBERG ${ }^{3}$, DANIELA SIMIAN ${ }^{4, b}$, GONZALO PIZARRO ${ }^{1, \mathrm{c}}$, \\ PAOLA TOCHE ${ }^{5}$, RODRIGO QUERA ${ }^{2}$
}

\section{Natalizumab for the treatment of Crohn's disease. Report of three cases}

\begin{abstract}
Anti-tumor necrosis factor- $\alpha$ (TNF) agents have dramatically changed the management of Crohn's Disease (CD). However, a significant number of these patients do not respond at all or cease to respond to antibodies against TNF. In this clinical situation, the options include intensification of anti-TNF therapy by either increasing the dose or by shortening the administration interval, the use of a second anti-TNF or medications with a different mechanism of action. Among the later, Natalizumab, a humanized IgG4 monoclonal antibody against $\alpha 4 \beta 1$ and $\alpha 4 \beta 7$ integrins, is safe and effective in inducing and maintaining remission in active CD patient's refractory to anti-TNF. In spite of this, Natalizumab use has been limited because of an increased risk of progressive multifocal leukoencephalophaty which results from reactivation of the John Cunningham (JC) virus. However, the presence of antibodies against JC virus in serum can be used to reduce the risk for this complication. We report three patients with Crohn's disease refractory to treatment with infliximab, who responded successfully to the use of Natalizumab.
\end{abstract}

(Rev Med Chile 2017; 145: 538-543)

Key words: Biological Therapy; Crohn Disease; Inflammatory Bowel Disease; Natalizumab.
L a enfermedad de Crohn (EC) es una enfermedad inflamatoria crónica que puede comprometer cualquier área del aparato digestivo y que se caracteriza por períodos de exacerbación y remisión afectando finalmente la calidad de vida ${ }^{1}$. Aunque la incidencia y prevalencia de la EC en Chile es aún desconocida, recientemente demostramos que el número de casos ha aumentado durante las últimas décadas ${ }^{2}$. Los objetivos terapéuticos son disminuir la inflamación e inducir la remisión clínica, endoscópica y radiológica de la enfermedad. Para lograr esto, la estrategia más efectiva es el uso de terapia biológica (TB) dado su rol en neutralizar vías inmunológicas específicas involucradas en el desarrollo y evolución de la EC. En la última década, los anticuerpos anti-factor de necrosis tumoral- $\alpha$ (anti-TNF) (infliximab, adalimumab y certolizumab) han sido la TB más utilizada en lograr la inducción y mantención de la remisión en pacientes con EC moderada-severa ${ }^{2,3}$. Sin embargo, aproximadamente $30-40 \%$ de los pacientes no responderán a la terapia de inducción (no-respondedores primarios) y hasta un 50\% perderán respuesta durante la fase de mantención (no-respondedores secundarios) siendo necesario intensificar el tratamiento (aumentar la dosis o 
acortar la frecuencia de administración del fármaco), cambiar de anti-TNF o de tipo de biológico ${ }^{4,5}$. En este último escenario, las posibilidades son el uso de anticuerpos anti-integrinas (natalizumab, vedolizumab $)^{6} \mathrm{o}$ anticuerpos anti-subunidad $\mathrm{p} 40$ de la interleukina-12/23 (ustekinumab) ${ }^{7}$.

Natalizumab es un anticuerpo monoclonal IgG4 anti-integrina- $\alpha 4$ humanizado que bloquea la adhesión y migración de leucocitos desde los vasos sanguíneos al tejido inflamado ${ }^{7,8}$.

Este fármaco ha mostrado ser efectivo en inducir y mantener la remisión en pacientes con $\mathrm{EC}^{9-18}$. Sin embargo, su uso se ha visto limitado por reportes que han descrito el desarrollo de leucoencefalopatía multifocal progresiva (LMP), infección oportunista fatal causada por la reactivación del virus latente John Cunningham (JC), en pacientes con EC y esclerosis múltiple (EM) tratados con natalizumab ${ }^{19,20}$. El objetivo de nuestro artículo es mostrar a través de tres casos la seguridad y efectividad de natalizumab en el tratamiento de la EC refractaria a TB anti-TNF.

\section{Casos clínicos}

Las características demográficas y clínicas se muestran en la Tabla 1.

\section{Paciente $N^{\circ} 1$}

Diagnosticado inicialmente con Colitis Ulcerosa el 2012, sin respuesta a mesalazina ni azatioprina. Inicia infliximab con respuesta inicial por 4 meses por lo que se cambia a adalimumab, presentando respuesta parcial pese a optimización con administración semanal. Evoluciona con diarrea con sangre realizándose una cápsula endoscópica que muestra actividad inflamatoria a nivel de íleon cambiándose el diagnóstico a EC. Se inicia natalizumab, estudio serológico para virus JC negativo. En contexto de alteración de pruebas hepáticas se realiza colangioresonancia que muestra dilatación de la vía biliar intrahepática y zonas de estenosis sugerente de Colangitis Esclerosante Primaria. Actualmente y tras 9 dosis de natalizumab, la paciente se encuentra asintomática con un índice

Tabla 1. Características demográficas y clínicas de los pacientes

\begin{tabular}{|c|c|c|c|}
\hline & Paciente $\mathbf{N}^{\circ} \mathbf{1}$ & Paciente $\mathbf{N}^{\circ} \mathbf{2}$ & Paciente $\mathbf{N}^{\circ} \mathbf{3}$ \\
\hline Sexo & Femenino & Masculino & Femenino \\
\hline Edad & 52 & 49 & 44 \\
\hline Tabaco & No & No & No \\
\hline Años de enfermedad & 4 & 5 & 6 \\
\hline Localización & Ileocolónica & Colon & Colon \\
\hline Fenotipo & Inflamatorio & Inflamatorio & Inflamatorio + Perianal \\
\hline Manifestaciones extraintestinales & Colangitis esclerosante & Pancreatitis autoinmune & Articular \\
\hline Terapias previas & $\begin{array}{l}5-A S A \\
\text { CTC } \\
\text { AZA } \\
\text { IFX }(5 \mathrm{mg} / \mathrm{Kg}) \\
\text { ADA }\end{array}$ & $\begin{array}{l}\text { 5-ASA } \\
\text { CTC } \\
\text { MTX } \\
\text { IFX }(5 \mathrm{k} \mathrm{mg} / \mathrm{Kg})\end{array}$ & $\begin{array}{l}\text { 5-ASA } \\
\text { CTC } \\
\text { Ciprofloxacino/Metronidazol } \\
\text { AZA } \\
\text { IFX }(5 \mathrm{mg} / \mathrm{Kg}-10 \mathrm{mg} / \mathrm{Kg})\end{array}$ \\
\hline Hospitalizaciones & 0 & 1 & 1 \\
\hline Cirugías & No & No & $\begin{array}{l}\text { Sí (Fístula) } \\
\text { Setón (2) } \\
\text { Trasplante células madre }\end{array}$ \\
\hline Dosis Natalizumab & 9 & 8 & 14 \\
\hline SES-CD al inicio & - & 13 & 24 \\
\hline SES-CD control & - & 3 & 0 \\
\hline
\end{tabular}

5-ASA $=5$ Aminosalicilatos, $C T C=$ esteroides, AZA = Azatioprina, MTX = Metotrexato, IFX = Infliximab, ADA = Adalimumab. 
Harvey-Bradshaw 0 y control con enterografía por resonancia (e-RM) de abdomen-pelvis que muestra discreto engrosamiento parietal del íleon terminal de carácter inespecífico.

\section{Paciente $N^{\circ} 2$}

Diagnosticado con EC el 2011, inicialmente tratado sólo con mesalazina. El 2014 desarrolla pancreatitis autoinmune, posteriormente evoluciona con compromiso del estado general (CEG), baja de peso de $12 \mathrm{~kg}$, anemia y deposiciones líquidas con sangre. El estudio colonoscópico y la e-RM de abdomen-pelvis mostraron severo compromiso inflamatorio segmentario del colon. Se inicia infliximab y metotrexato. Posterior a $3^{\text {a }}$ dosis de infliximab presenta reactivación con deposiciones con sangre y estudio que confirma actividad inflamatoria moderada a nivel de colon (SES-CD 13) (Figura 1A-B-C). Se inicia natalizumab, estudio serológico para virus JC negativo. $\mathrm{Al}$ recibir la $2^{\mathrm{a}}$ dosis presenta rash cutáneo y dificultad respiratoria leve que ceden con la administración de esteroides y suspensión del fármaco. Se realiza desensibilización con protocolo de tolerancia permitiendo la reintroducción del fármaco sin inconvenientes. Actualmente y tras
8 dosis de natalizumab, el paciente se encuentra asintomático con un índice Harvey-Bradshaw 0 y una colonoscopía con SES-CD de 3 (Figura 1D-E-F).

\section{Paciente $N^{\circ} 3$}

Diagnosticada con EC el 2010 con compromiso inflamatorio de colon y enfermedad perianal refractaria a azatioprina. Inicia el 2011 infliximab con respuesta clínica favorable hasta septiembre de 2012 donde presenta reactivación de su enfermedad con compromiso inflamatorio de colon-recto y una fístula perianal aumentándose la dosis de infliximab a $10 \mathrm{mg} / \mathrm{kg}$ cada 8 semanas y se instala sedal. En agosto de 2013 se realiza tratamiento con células madres en trayecto fistuloso. Se mantiene estable hasta enero de 2015 donde refiere CEG, baja de peso, dolor abdominal y deposiciones líquidas con sangre. La colonoscopía mostró compromiso severo del colon (SES-CD 24) (Figura 2A-B-C). Se inicia natalizumab, estudio serológico para virus JC negativo. Actualmente y tras 14 dosis de natalizumab, la paciente se encuentra asintomática con un índice Harvey-Bradshaw 0 y una colonoscopía con un SES-CD de 0 (Figura 2D-E-F).
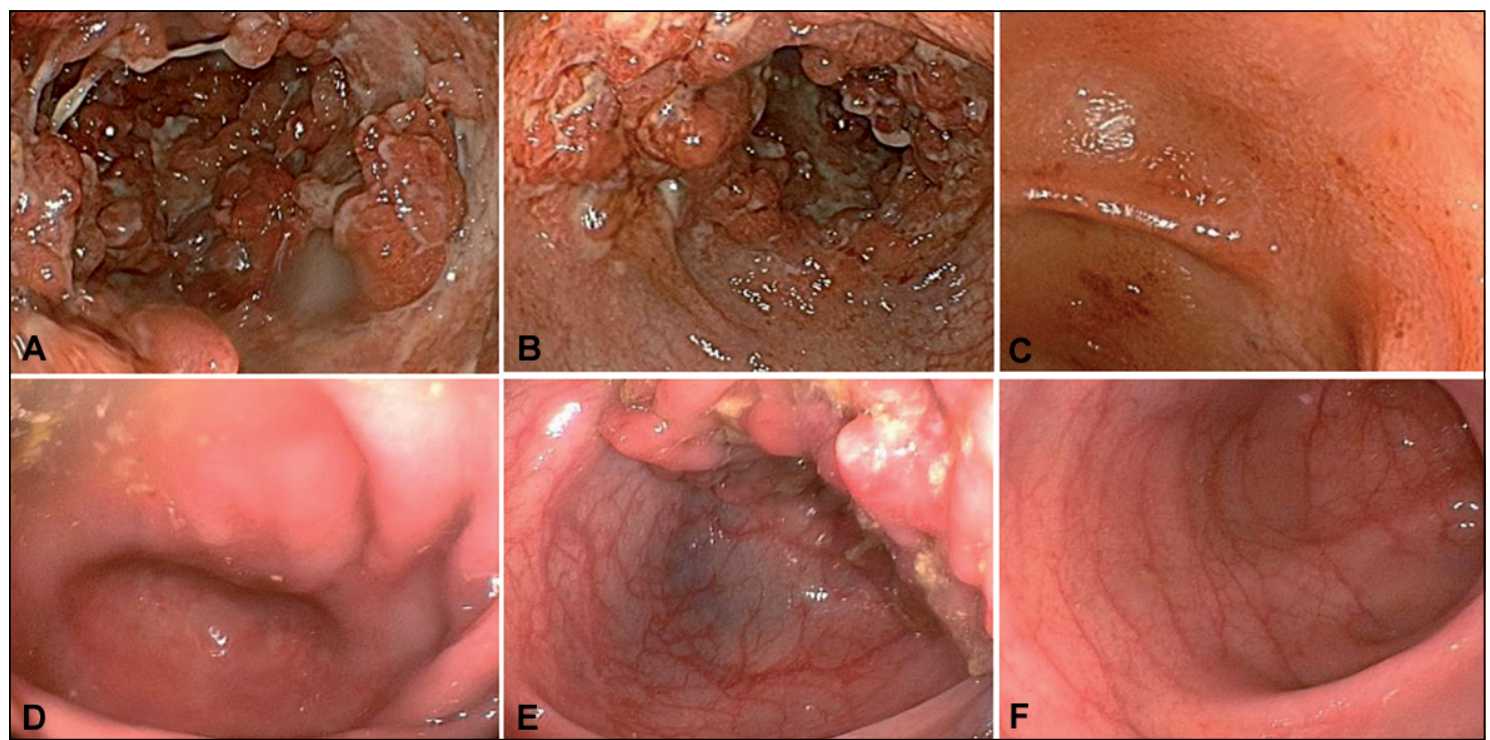

Figura 1. Colonoscopias paciente 2. A-B-C: imágenes colonoscopia previo al uso de natalizumab. A-B. Pólipos inflamatorios y úlceras en ciego y colon derecho respectivamente. C. Erosiones en recto. D-E-F: imágenes colonoscopia posterior al uso de natalizumab. D. Colon ascendente sano. E. Pólipos inflamatorios en colon ascendente. F. Colon descendente sano. 


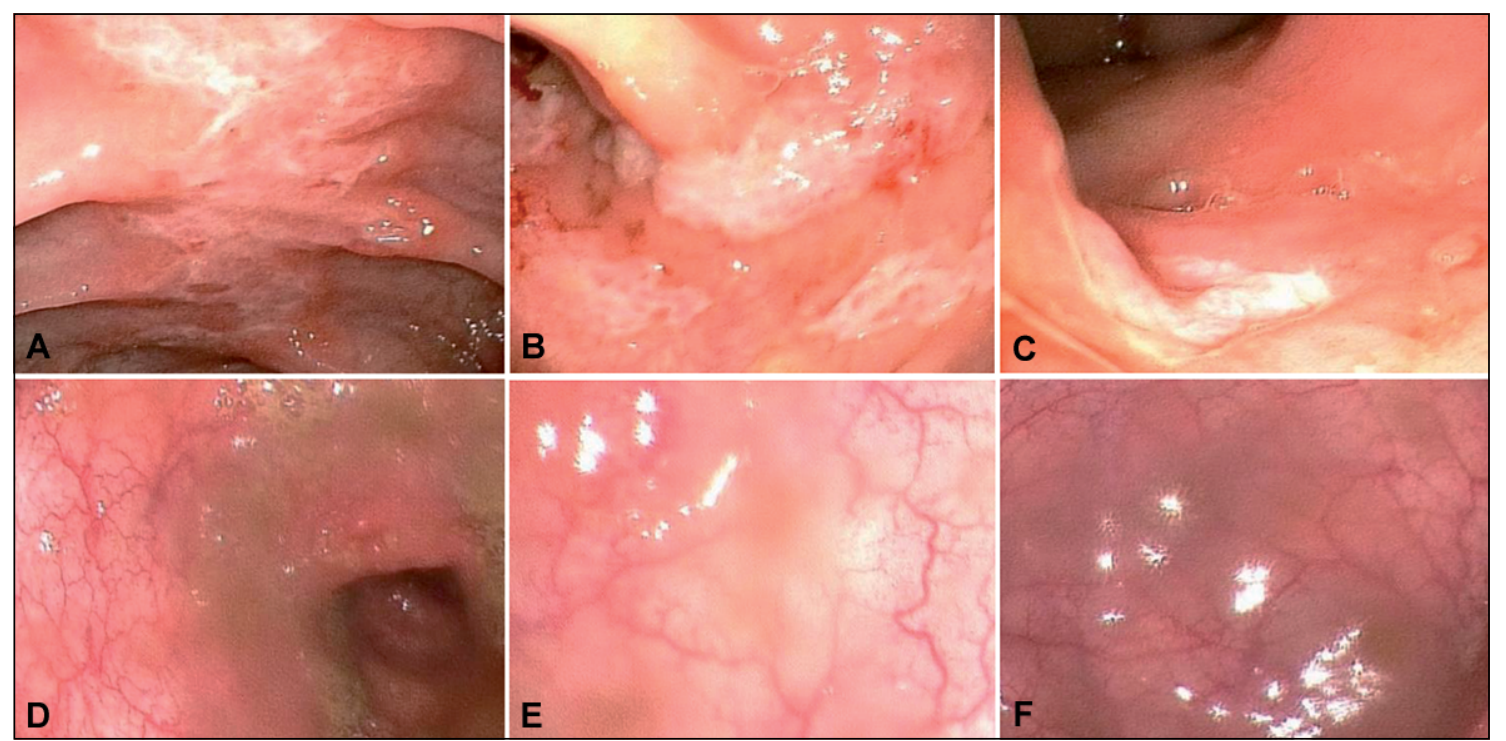

Figura 2. Colonoscopias paciente 3. A-B-C: imágenes colonoscopia previo al uso de natalizumab. A. Úlcera colon transverso. B. Úlceras sigmoides. C. Úlcera recto. D-E-F: imágenes colonoscopia posterior al uso de natalizumab. D. Colon sano. E. Sigmoides sano. F. Recto sano.

\section{Discusión}

La inhibición del tráfico de leucocitos hacia la mucosa intestinal durante el proceso de inflamación ha resultado ser el segundo blanco terapéutico en el desarrollo de TB para el manejo de la Enfermedad Inflamatoria Intestinal. Natalizumab y vedolizumab han sido aprobados en el uso de la EC moderada-severa, especialmente en aquellos pacientes que no responden a la fase de inducción o desarrollan pérdida de respuesta durante la terapia de mantención. Vedolizumab, a diferencia de natalizumab, es una anti-integrina $\alpha 4 \beta 7$ sin actividad contra la integrina $\alpha 4 \beta 1$, haciendo que el riesgo de LMP sea extremadamente bajo, principalmente debido a que no atraviesa la barrera hematoencefálica ${ }^{6}$. Esto hace que vedolizumab sea la primera elección al decidir el uso de una TB anti-integrina. Sin embargo, este fármaco no está actualmente disponible en nuestro país, siendo natalizumab la única anti-integrina en EC refractaria a TB anti-TNF.

Natalizumab usado como monoterapia en dosis de $300 \mathrm{mg}$ cada 4 semanas ha demostrado ser efectivo y seguro en EC refractaria a agentes anti-TNF ${ }^{-17}$. Además, este fármaco ha demostrado ser eficaz en población pediátrica con EC con tasas de remisión clínica de $50 \%{ }^{18}$. Otros han señalado que su uso puede disminuir el riesgo de cirugía ${ }^{21}$ y mejorar la calidad de vida de los pacientes con $\mathrm{EC}^{22}$. Nuestra experiencia demuestra que natalizumab tuvo una respuesta efectiva alcanzando la remisión clínica en los tres pacientes. Sakuraba y cols., demostraron en un estudio retrospectivo que la tasa de respuesta es menor en pacientes con SES-CD severo y que la presencia de curación mucosa era predictor de una respuesta prolongada durante la fase de mantención ${ }^{23}$. Dos de nuestros pacientes presentaron una respuesta endoscópica, logrando uno de ellos una disminución del SES-CD $>70 \%$ y el otro un SES-CD de 0. El tercer paciente presentó una respuesta radiológica adecuada tras 9 dosis de natalizumab con presencia de discreto engrosamiento parietal del íleon terminal de carácter inespecífico en una e-RM.

En nuestro estudio, los tres pacientes tuvieron serología negativa para el virus JC previo al inicio de natalizumab. Estudios han demostrado que la prevalencia de exposición al virus JC en pacientes con EC es similar a la población general ${ }^{21}$. El uso de tiopurínicos, a diferencia de metotrexato, sería un factor de riesgo de tener una serología positiva ${ }^{21}$. Dos de nuestros pacientes utilizaron azatioprina 
previo al uso de natalizumab. Scott y cols., utilizaron un Modelo Markov para evaluar el impacto que tendría la presencia del virus JC demostrando que en pacientes con serología negativa el uso de natalizumab sería la estrategia más costo efectiva versus utilizar un segundo anti-TNF ${ }^{24}$.

La mayoría de los reportes sobre LMP asociado a natalizumab provienen de pacientes con EM. Entre 99.571 pacientes con EM tratados con natalizumab, se reportaron 212 casos de LMP $(2,1 / 1.000)^{25}$. La incidencia de LMP fue de 0,09 por 1.000 en pacientes con anticuerpos virus JC negativo, mientras en esos con anticuerpos positivo, el uso previo de inmunosupresores y una duración de natalizumab de más de dos años aumento el riesgo a 11,1/1.000. La tasa de conversión en pacientes con virus JC negativo es extremadamente baja, lo que permitiría el uso de natalizumab por un período prolongado. Se ha sugerido que este grupo de pacientes sea controlado cada 6-12 meses para confirmar que mantienen anticuerpos negativos $^{21}$. Nuestros tres pacientes han sido chequeados a los 6 y 12 meses manteniendo anticuerpos negativos para el virus JC.

Un metaanálisis reciente que incluyó 12 estudios randomizados y controlados con más de 3.000 pacientes tratados con anti-integrinas no demostró un aumento significativo en el riesgo de infecciones oportunistas, neoplasia intestinal o extraintestinal al compararlo con placebo ${ }^{26}$. Dos metaanálisis del mismo año confirman la seguridad de natalizumab y vedolizumab en pacientes con $\mathrm{EC}^{27,28}$. Una reciente nota informativa de farmacovigilancia nacional señala que se han registrado 5 notificaciones de reacciones adversas asociadas a natalizumab, ninguna correspondiendo a LMP(disponible en: http://www.ispch.cl/sites/default/files/comunicado/2016/06/NOTA\%20 INFORMATIVA\%20DE\%20FARMACOVIGILANCIA,\%20LEUCOENCEFALOPAT\%C3\%8DA.pdf). Uno de nuestros pacientes presentó un evento adverso leve caracterizado por compromiso cutáneo y respiratorio siendo manejado con esteroides y desensibilización. Protocolos de tolerancia en caso de hipersensibilidad a natalizumab han demostrado ser efectivos permitiendo la reintroducción y mantención del fármaco ${ }^{29}$.

En conclusión, aunque vedolizumab con su mejor perfil de seguridad es la primera indicación al momento de optar por una TB anti-integrina, la literatura y nuestra experiencia muestran que natalizumab es una opción viable en pacientes con EC refractaria a anti-TNF o que hayan presentado eventos adversos serios a estos fármacos.

\section{Referencias}

1. Simian D, Quera R. Manejo integral de la enfermedad inflamatoria intestinal: más allá de una terapia farmacológica adecuada. Rev Med Chile 2016; 144: 488-95.

2. Simian D, Fluxá D, Flores L, Lubascher J, Ibáñez P, Figueroa $\mathrm{C}$, et al. Inflammatory Bowel disease: A descriptive study of 716 local Chilean patients. World J Gastroenterol 2016; 22: 5267-75.

3. Terdiman JP, Gruss CB, Heidelbaugh JJ, Sultan S, Falck-Ytter YT, AGA Institute Clinical Practice and Quality Management Committee. American Gastroenterology Association Institute guideline on the use of thiopurines, methotrexate, and anti-TNF- $\alpha$ biological drugs for the induction and maintenance of remission in inflammatory Crohn's disease. Gastroenterology 2013; 145: 1459-63.

4. Yanai H, Hanauer SB. Assessing response and loss of response to biological therapies in IBD. Am J Gastroenterol 2011; 106: 685-98.

5. Strik AS, Bots SJ, D'Haens G, Löwenberg M. Optimization of anti-TNF therapy in patients with Inflammatory Bowel Disease. Expert Rev Clin Pharmacol 2016; 9: 42939.

6. Khanna R, Mosli MH, Feagan B. Anti-integrins in Ulcerative Colitis and Crohn's disease: What is their place? Dig Dis 2016; 34: 153-9.

7. Engel T, Kopylov U. Ustekinumab in Crohn's disease: evidence to date and place in therapy. Ther Adv Chronic Dis 2016; 7: 208-14.

8. Binion DG, West GA, Ina K, Ziats NP, Emancipator SN, Fiocchi C. Enhanced leukocyte binding by intestinal microvascular endothelial cells in inflammatory bowel disease. Gastroenterology 1997; 112: 1895-907.

9. Gordon FH, Lai CW, Hamilton MI, Allison MC, Srivastava ED, Fouweather MG, et al. A randomized placebo-controlled trial of a humanized monoclonal antibody to alpha4 integrin in active Crohn's disease. Gastroenterology 2001; 121: 268-74.

10. Ghosh S, Goldan E, Gordon FH, Malchow HA, RaskMadsen J, Rutgeerts P, et al. Natalizumab for active Crohn's disease. N Engl J Med 2003; 348: 24-32.

11. Sandborn WJ, Colombel JF, Enns R, Feagan BG, Hanauer SB, Lawrance IC, et al. Natalizumab induction and maintenance therapy for Crohn's disease. N Engl J Med 2005; 353: 1912-25.

12. Sands BE, Kozarek R, Spainhour J, Barish CF, Becker S, 
Goldberg L. Safety and tolerability of concurrent natalizumab treatment for patients with Crohn's disease not in remission while receiving infliximab. Inflamm Bowel Dis 2007; 13: 2-11.

13. Targan SR, Feagan BG, Fedorak RN, Lashner BA, Panaccione R, Present DH, et al. Natalizumab for the treatment of active Crohn's disease: results of the ENCORE Trial. Gastroenterology 2007; 132: 1672-83.

14. Kane SV, Horst S, Sandborn WJ, Becker B, Neis B, Moscandrew $\mathrm{M}$, et al. Natalizumab for moderate to severe Crohn's disease in Clinical practice: The Mayo Clinic Rochester Experience. Inflamm Bowel Dis 2012; 18: 2203-8.

15. Chen $\mathrm{CH}$, Kularatna G, Stone CD, Gutiérrez AM, Dassopoulos T. Clinical experience of natalizumab in Crohn's disease patients in a restricted distribution program. Ann Gastroenterol 2013; 26: 233-8.

16. Sakuraba A, Keyashian K, Correia C, Melek J, Cohen RS, Hanauer SB, et al. Natalizumab in Crohn's disease: results from a US tertiary inflammatory bowel disease center. Inflamm Bowel Dis 2013; 19: 621-6.

17. Juillerat P, Wasan S, Fowler SA, Friedman S, Pabby VK, Coukas JA, et al. Efficay and safety of Natalizumab ib Crohn's disease patients treated a 6 Bosotn Academic Hospitals. Inflamm Bowel Dis 2013; 19: 2457.63.

18. Singh N, Deshpande R, Rabizadeh S, Dubinsky M. Real world experience with Natalizumab at a tertiary care pediatric IBD center. JGPN. 2016; 62: 863-6.

19. Van Assche G, Van Ranst M, Sciot R, Dubois B, Vermeire S, Noman M, et al. Progressive multifocal leukoencephalopathy after natalizumab therapy in Crohn's disease. N Engl J Med 2005; 353: 362-8.

20. Langer-Gould A, Atlas SW, Green AJ, Bollen AW, Pelletier D. Progressive multifocal leukoencephalopathy in a patient treated with natalizumab. N Engl J Med 2005; 353: 375-81.

21. Bellaguarda E, Keyashian K, Pekow J, Rubin DT, Cohen $\mathrm{RD}$, Sakuraba A. Prevalence of antibodies against JV virus in patients with refractory Crohn's disease and effects of natalizumab therapy. Clin Gastroenterol Hepatol 2015; 13: 1919-25.

22. Dudley-Brown S, Nag A, Cullinan C, Ayers M, Hass S, Panjabi S. Health-related quality-of-life evaluation of Crohn's disease patients after receiving natalizumab therapy. Gastroenterol Nurs 2009; 32: 327-59.

23. Sakuraba A, Annunziata ML, Cohen RD, Hanauer SB, Rubin DT. Mucosal healing is associated with improved long-term outcome of maintenance therapy with natalizumab in Crohn's disease. Inflamm Bowel Dis 2013; 19: 2577-83.

24. Scott FI, Osterman MT, McConnell RA, Lorusso M, Aberra F, Kerner C, et al. Impact of JC virus antibody testing in patients with Crohn's disease with loss of response to infliximab: A Markov Model. Inflamm Bowel Dis 2013; 19: 2625-33.

25. Bloomgren G, Richman S, Hotermans C, Subramanyam M, Goelz S, Natarajan A, et al. Risk of natalizumab-associated progressive multifocal leukoencephalophaty. N Engl J Med 2012; 366: 1870-80.

26. Luthra P, Peyrin-Biroulet L, Ford AC. Systematic review and meta-analysis: opportunistic infections and malignancies during treatment with anti-integrin antibodies in inflammatory bowel disease. Aliment Pharmacol Ther 2015; 41: 1227-36.

27. Chandar AK, Singh S, Murad MH, Peyrin-Biroulet L, Loftus EV. Efficacy and safety of natalizumab and vedolizumab for the management of Crohn's disease: A systematic review and meta-analysis. Inflamm Bowel Dis 2015; 21: 1695-708.

28. Ge WS, Fan JG. Integrin antagonists are effective and safe for Crohn's disease: A meta-analysis. World J Gastroenterol 2015; 21: 4744-9.

29. Camacho-Halili M, George R, Gottesman M, Davis-Lorton $\mathrm{M}$. An approach to natalizumab hypersensitivity a case series of induction of tolerance. Mutl Scler 2011; 17: 250-3. 